\title{
Correspondence
}

\section{Anaphylactic reactions}

To the Editor:

To evaluate patients with suspected drug-induced anaphylactic reactions during anaesthesia, we are currently studying a new test for mast-cell activation that should be helpful in investigating these life-threatening reactions. If you have a patient who has developed a suspected anaphylactic reaction, please obtain one serum or plasma sample as soon as possible during the reaction, then an additional sample one hour later, and forward to me with the anaesthesia records. If your have any questions, please also call 404-248-3203. Thank you for your co-operation.

Jerrold H. Levy MD

Department of Anesthesiology

Emory University School of Medicine

1364 Clifton Rd, N.E.

Atlanta, Georgia 30322

\section{Intrapleural block}

To the Editor:

I draw your attention to the recent letter "Bilateral interpleural block for midline upper abdominal surgery." ' As the pleura is an organ paired about the midline I cannot conceive how "interpleural" block can be bilateral. This cystic organ has been invaginated causing apparent layering of its wall, and improper use of synecdoche has led us to speak of two pleurae on either side, visceral and parietal. Intrapleural is a preferred term for what the author intends. However, let us cultivate simplicity and refer to pleural block, left and/or right. We will thus gain in clarity, succinctness and accuracy.

\section{G. Philip Anthony MB FRCPC \\ 308 Dromore Avenue \\ Winnipeg, R3M 0J5}

\section{REFERENCE}

1 Lee $E$. Bilateral interpleural block for midline upper abdominal surgery. Can J Anaesth 1991; 38: 683.

\section{Intrapleural epidural catheter for the treatment of pneumothorax}

To the Editor:

Intercostal blockade is an accepted method of postoperative pain relief after cholecystectomy operations and pneumothorax is a recognised complication of this block. During the past year, three patients developed pneumothorax, with symptoms, following intercostal blockade. In each case an epidural catheter was sited intrapleurally at the sixth intercostal space in the mid-axillary line and the pneumothorax could be treated by aspirating the air and evacuating it through a three-way stopcock. The catheters were removed after $24 \mathrm{hr}$, when no more air could be aspirated. Furthermore the catheters were used to provide intrapleural analgesia for postoperative pain relief in these patients. $^{1}$

\section{V.J. Sarma FFARCSI \\ Piteå General Hospital \\ 94128 Piteå, Sweden}

\section{REFERENCE}

1 Reiestad F, Stromskag KE. Interpleural catheter in the management of postoperative pain. Regional Anesthesia 1986; 11: 89-91.

\section{Obstetrical anaesthesia for patients with HELLP syndrome}

\section{To the Editor:}

The review article by Crosby regarding the anaesthetic management of patients with HELLP syndrome ${ }^{1}$ was both interesting and informative. His advice as to the place of epidural anaesthesia in this condition was well reasoned but I would like to add two further points.

First, in the patient who is at high risk of developing a coagulopathy it would seem sensible to reduce the incidence of epidural vessel puncture. As the epidural veins are situated along the lateral walls of the epidural space, ${ }^{2}$ a midline approach will decrease this risk, as will the 
threading of only $3-4 \mathrm{~cm}$ of catheter ${ }^{3}$ and the previous injection of bupivacaine through the epidural needle. ${ }^{4}$

Crosby advises that it may be prudent to remove the catheter as soon as possible after delivery. If the epidural has been placed for vaginal delivery, the patient may have developed a coagulopathy with the catheter in situ. To my knowledge, there is no objective evidence as to whether the catheter should be removed or left in the space in this situation. Rao and EL-Etr ${ }^{5}$ favour leaving the catheter until the coagulopathy has resolved and this advice is echoed by Stanley and Lunn. ${ }^{6}$ Their recommendations follow therapeutic heparin administration and the situation of a rapidly developing coagulopathy in the context of HELLP syndrome may be different.

However, one could envisage that if there were epidural venous trauma on insertion of the catheter, its subsequent removal in the presence of a coagulopathy might dislodge a clot and lead to an expanding haematoma with disastrous consequences.

More information is needed, but my present practice is to leave the epidural catheter in position until any coagulopathy has resolved. It would be interesting to know if this practice is followed by your readership.

Peter D. Collins MB FCAnaesth.

RGH Hollywood,

Nedlands

W. Australia

\section{REFERENCES}

1 Crosby ET. Obstetrical anaesthesia for patients with the syndrome of haemolysis, elevated liver enzymes and low platelets. Can J Anaesth 1991; 38: 227-33.

2 Gillian LA. Veins of the spinal cord. Anatomic details; suggested clinical applications. Neurology 1970;20: 860-8.

3 Odoom JA, Sih IL. Epidural analgesia and anticoagulant therapy. Experience with one thousand cases of continuous epidurals. Anacsthesia 1983; 38: 254-9.

4 Verniquet $A J W$. Vessel puncture with epidural catheters. Experience in obstetric patients. Anaesthesia 1980; 35: 660-2.

5 Rao $T L K, E L-E t r A A$. Anticoagulation following placement of epidural and subarachnoid catheters: an evaluation of neurologic sequelae. Anesthesiology 1981; 55: 618-20.

6 Stanley TH, Lunn JK. Anticoagulants and continuous epidural anaesthesia (letter). Anesth Analg 1980; 59: 394-5.

\section{REPLY}

I would like to thank Dr. Collins for his interest in the review and his comments regarding the use of epidural anaesthesia in this patient population. I would suggest that, in patients at high risk of developing a consumptive coagulopathy, it would seem prudent to limit the use of epidural blockade. Careful assessment of the risks and benefits is emphasized and I would suspect that, the as yet undefined risk of bleeding into the epidural space probably outweighs the apparent benefits of epidural blockade in the majority of patients with HELLP syndrome. The incidence of epidural blood vessel trauma has recently been estimated to be as high as $12 \%$ but I am not aware of data that suggest or confirm that the paramedian approach to the epidural space is associated with a higher incidence of vessel trauma.' Although lagree with the recommendation for threading the catheter 3-4 $\mathrm{cm}$ only, it is because the incidence of unsatisfactory block appears to increase as excess length of carheter is placed into the epidural space. Again, I am not aware of data that confirm a higher incidence of vessel trauma with increasing length of catheter thread into the epidural space. Although injection of 10 $\mathrm{ml}$ of bupivacaine through the needle before passing the catheter has been shown to decrease the incidence of vessel trauma from $9 \%$ to $3 \%$, this technique would result in total spinal anaesthesia if accidentally injected intrathecally or local anaesthetic toxicity if injected intravascularly. ${ }^{2}$ Smaller volumes of local anaesthetic or saline have not been demonstrated to be efficacious in reducing the incidence of vessel trauma. ${ }^{3}$ Larger volumes of saline could be employed but the diluting effect on the subsequently injected local anaesthetic would have to be considered.

With respect to the advisability of removing the catheter and the optimum time to do so, opinions vary. We are in agreement that, with respect to an epidural catheter placed before heparin anticoagulation, there seems to be little rationale to removing the catheter during the transient period of controlled anticoagulation. However, the scenario of the patient with HELLP syndrome and a consumptive coagulopathy that is usually shortlived but that may persist for days may not parallel that of the heparinized patient. If the diagnosis of HELLP syndrome was made before there was evidence of clinical coagulopathy, then I would remove the catheter immediately postpartum. If there was clinical evidence of coagulopathy, then my decision whether to remove the catheter or not would be influenced by whether or not there was an obvious therapeutic benefit to maintaining the epidural analgesia. That is, I would maintain the catheter in situ if I was going to use it. It is well documented that epidural catheters do migrate in a large proportion of patients and both inward migration with latent blood vessel and dural puncture and catheter migration out of the space do occur. ${ }^{4.5}$ Although it is speculative, this uncontrolled catheter movement may represent a greater risk of aggravating previous vessel trauma or initiating new trauma than a careful and controlled removal of the catheter from the epidural space. Therefore, if I had no plans to use the catheter I would remove it.

E.T. Crosby BSc MD FRCPC

Ottawa

\section{REFERENCES}

1 Rolbin SH, Hew E. A comparison of two types of epidural catheters. Can J Anaesth 1987; 34: 459-61.

2 Verniquet $A J W$. Vessel puncture with epidural catheters. Anaesthesia 1980; 35: 660-2.

3 Rolbin SH, Halpern SH, Braude BM, Kapala D, Unger R, Radhakrisnan $S$. Fluid through the epidural needle does not reduce complications of epidural catheter insertion. Can J Anaesth 1990; 37: 337-40. 